\title{
Accuracy of Noninvasive Scoring Systems in Assessing Liver Fibrosis in Patients with Nonalcoholic Fatty Liver Disease: A Systematic Review and Meta-Analysis
}

\author{
Sangsoo Han ${ }^{1}$, Miyoung Choi ${ }^{2}$, Bora Lee ${ }^{3}$, Hye-Won Lee ${ }^{4}$, Seong Hee Kang ${ }^{5}$, Yuri Cho ${ }^{6}$, Sang Bong Ahn ${ }^{7}$, Do \\ Seon Song ${ }^{8}$, Dae Won Jun', Jieun Lee ${ }^{10}$, and Jeong-Ju Yoo ${ }^{11}$ \\ ${ }^{1}$ Department of Emergency Medicine, Soonchunhyang University Bucheon Hospital, Bucheon, ${ }^{2}$ Clinical Evidence Research, National \\ Evidence-based Healthcare Collaborating Agency (NECA), ${ }^{3}$ Department of Statistics, Graduate School of Chung-Ang University, \\ ${ }^{4}$ Department of Internal Medicine, Yonsei University College of Medicine, Seoul, ${ }^{5}$ Department of Internal Medicine, Wonju Severance \\ Christian Hospital, Yonsei University Wonju College of Medicine, Wonju, ${ }^{6}$ Center for Liver and Pancreatobiliary Cancer, National \\ Cancer Center, Goyang, ' Department of Internal Medicine, Nowon Eulji Medical Center, Eulji University College of Medicine, Seoul, \\ ${ }^{8}$ Department of Internal Medicine, St. Vincent's Hospital, College of Medicine, The Catholic University of Korea, Suwon, ${ }^{9}$ Department \\ of Internal Medicine, Hanyang University College of Medicine, Seoul, ${ }^{10}$ Department of Internal Medicine, Soonchunhyang University \\ College of Medicine, Cheonan, and ${ }^{11}$ Department of Internal Medicine, Soonchunhyang University Bucheon Hospital, Bucheon, Korea
}

\section{Article Info}

Received August 24, 2021

Revised November 18, 2021

Accepted November 23, 2021

Published online February 23, 2022

\section{Corresponding Author}

Dae Won Jun

ORCID https://orcid.org/0000-0002-2875-6139

E-mail noshin@hanyang.ac.kr

Jeong-Ju Yoo

ORCID https://orcid.org/0000-0002-7802-0381

E-mail puby17@naver.comr

Sangsoo Han and Miyoung Choi contributed equally to this work as first authors.
Background/Aims: Several noninvasive scoring systems have been developed to determine the risk of advanced fibrosis in nonalcoholic fatty liver disease (NAFLD). We examined the diagnostic accuracy of the fibrosis-4 (FIB-4) score and NAFLD fibrosis score (NFS) in patients with biopsy-proven NAFLD.

Methods: For this meta-analysis, various databases including PubMed (MEDLINE), EMBASE, OVID Medline and the Cochrane Library were systematically searched. After the acquired abstracts were reviewed by two investigators, manuscripts were chosen for a full-text examination.

Results: Thirty-six studies evaluating biopsy-proven NAFLD were selected for meta-analysis. A total of 14,992 patients were analyzed. The lower cutoff sensitivity of the FIB-4 score predicting histological fibrosis stage 3 or more $(\geq F 3)$ was $69 \%$, with specificity of $64 \%$, positive likelihood ratio (LR+) of 1.96, and negative likelihood ratio (LR-) of 0.47 . The low baseline sensitivity of the NFS score predicting $\geq F 3$ was $70 \%$, with a specificity of $61 \%$, LR + of 1.83 , and LR- of 0.48 . The area under the receiver operating characteristic curve (AUC) values of the FIB-4 score predicting $\geq \mathrm{F} 3$ and $\geq \mathrm{F} 2$ were $76 \%$ and $68 \%$, respectively. The AUC values of the NFS score predicting $\geq \mathrm{F} 3$ and $\geq \mathrm{F} 2$ were $74 \%$ and $60 \%$, respectively.

Conclusions: The FIB-4 or NFS test can be used to predict the degree of liver fibrosis in NAFLD, and the diagnostic accuracy resulted as relatively high in fibrosis stages of F3 or higher. (Gut Liver 2022;16:952-963)

Key Words: Liver fibrosis; Meta-analysis; Nonalcoholic fatty liver disease; Predictive value of tests

\section{INTRODUCTION}

With a prevalence of $25 \%$ to $40 \%$ in the general population, nonalcoholic fatty liver disease (NAFLD) is the most common liver disease worldwide, a pressing health concern associated with insulin resistance and metabolic syndrome. ${ }^{1,2}$ NAFLD affects nearly 100 million individu- als in the United States and occurs in 90\% of the obese population. ${ }^{1,3}$ Due to such burden of the disease, the early identification of patients with high morbidity and mortality associated with NAFLD is essential.

NAFLD can be categorized into various stages, from simple steatosis without fibrosis to nonalcoholic steatohepatitis related cirrhosis. ${ }^{1}$ The severity of NAFLD is 
determined by three factors: steatosis, inflammation, and fibrosis. Among these factors, the degree of hepatic fibrosis is the most essential factor in clinical settings, allowing clinicians to estimate the long-term prognosis in patients with NAFLD, such as the development of hepatocellular carcinoma, liver-related death or cardiovascular mortality. ${ }^{1,4}$ In fact, while simple steatosis is considered a nonprogressive condition, nonalcoholic steatohepatitis or significant fibrosis (SF) is regarded as one of the main causes of liver transplantation. ${ }^{5}$ Therefore, it is vital to promptly identify advanced fibrosis (AF; $\geq$ stage 3 fibrosis) or SF ( $\geq$ stage 2 fibrosis) in such patients. ${ }^{6}$

Liver biopsy is the gold standard for staging and identifying fibrosis in NAFLD patients. ${ }^{7}$ However, it is not suitable for a routine screening use, due to various reasons including its invasive nature, potential complications, possibility of sampling error, and high cost. ${ }^{7,8}$ Therefore, a simple, inexpensive and noninvasive panel to identify and quantify liver fibrosis is necessary. Likewise, though techniques such as magnetic resonance elastography or transient elastography have been recently developed, their high expense prevents their use as routine screening tests. Thus, noninvasive fibrosis scoring systems based on serologic tests such as the fibrosis-4 (FIB-4) index and NAFLD fibrosis score (NFS) have been developed and widely used as screening tools to assess the degree of fibrosis. ${ }^{9,10}$ However, a comprehensive study on such scoring system is crucial, since not only was the FIB-4 score developed only for patients with viral hepatitis, the accuracy of these serologic scoring systems in NAFLD patients also differ among studies. The aim of this systematic review and meta-analysis is to evaluate the diagnostic accuracy of noninvasive fibrosis scoring systems (FIB-4 and NFS), compared to that of the corresponding liver histologic data, to predict AF and SF in patients with NAFLD.

\section{MATERIALS AND METHODS}

This meta-analysis adhered to the protocol previously registered with PROSPERO (International Prospective Register of Systematic Reviews, CRD42021241243). We administered this systematic review and meta-analysis following guidelines provided by the Preferred Reporting Items for Systematic Reviews and Meta-Analyses extension for Diagnostic Test Accuracy.

\section{Inclusion criteria, exclusion criteria, and study outcome}

Studies that documented the accuracy of FIB-4 and NFS, evaluated by the corresponding liver histology results in NAFLD patients, were considered eligible for inclusion. The following criteria were required for studies to be selected: (1) patients with NAFLD; (2) reports of the accuracy of FIB-4 and NFS based on liver histology results. The state of fatty liver was determined by histologic characteristics. Eligible study designs were randomized controlled trials, cross-sectional studies, and cohort studies, both prospective and retrospective. Studies were excluded by the following criteria: (1) case reports; (2) case series, in which less than five patients in total were involved; (3) reviews; (4) cell or animal studies; (5) chronic viral hepatitis, such as hepatitis B or hepatitis C; (6) human immunodeficiency virus; (7) significant alcohol consumption; (8) fatty liver defined by imaging or serologic criteria, without any histology result provided; or (9) non-English studies.

The primary outcome of this meta-analysis was the diagnostic accuracy of the FIB-4 score and NFS, compared to the corresponding liver histology in patients with NAFLD.

\section{Search strategy}

We searched PubMed (MEDLINE), EMBASE, the Cochrane Library, Korean Medical Database, and Korean Studies Information Service System to identify studies published in English between January 1, 1997, and October 31, 2020. The keywords used in the Patient/Problem, Intervention, Comparison, and Outcome model are provided in the Supplementary Material. The search words were NAFLD index words, FIB-4-related index words or NFSrelated index words. We combined free-text words and controlled terms such as Medical Subject Headings and EMTREE according to the databases. The search strategy and the following result of each database are provided in the Supplementary Material. The entire search process was administered by a professional librarian (M.C.).

\section{Study selection and data extraction}

During the process of study selection, two reviewers (S.H. and J.J.Y.) first independently extracted relevant titles and abstracts. After an independent examination of the full-text articles, any resulting disparity between the two reviewers was resolved by a discussion with a third reviewer (H.W.L. or S.H.K.).

In addition, the two reviewers thoroughly examined the remaining procedures, such as screening full-text articles and assessing the risk of bias. The extraction of study characteristics and outcomes was conducted independently and documented in a standardized format by the two reviewers. Any discrepancy was settled by a discussion with Y.C. and S.B.A. 


\section{Methodological quality and risk of bias assessment}

To determine the risk of bias, we utilized the Cochrane risk of bias tool, with any relevant information provided in the Supplementary Material. Again, any discrepancy was resolved by a discussion with additional reviewers (D.S.S. and D.W.J.). Risk of bias was evaluated using two tools, QUADAS $^{11}$ and QUIPS ${ }^{12}$ tools. The overall outcome of the risk of bias is provided in the Supplementary Material. Publication bias was evaluated through a funnel plot.

\section{Statistical analysis}

The process of meta-analysis with sensitivity, specificity, positive likelihood ratio, and negative likelihood ratio is as the following: (1) transform the proportion into a quantity (Freeman-Tukey variant of the arcsine square root transformed the proportion); (2) calculate the pooled prevalence as the back-transformation of the weighted mean of the transformed prevalence using DerSimonian-Laird weights assuming the random-effect model; (3) calculate the confidence interval with the Clopper-Pearson interval. To further analyze the heterogeneity within the studies, researchers conducted a meta-regression to understand the influence of other factors on diagnostic accuracy. RevMan 5 (Cochrane Library) or the meta package in $\mathrm{R}$ version 4.1.0 (The R Foundation for Statistical Computing, Vienna, Austria) were utilized in the statistical analyses.

\section{RESULTS}

\section{Characteristics of included studies}

A thorough database search of titles and abstracts resulted in 86 relevant studies. Out of the 86,50 studies were excluded due to inappropriate patient population $(n=1)$, inappropriate outcome measurement $(n=42)$, overlapping population $(n=6)$, or insufficient data $(n=1)$. Finally, 36 studies were eligible for inclusion in this review (Fig. 1). Detailed characteristics of the studies included in this meta-analysis are provided in Table $1 .^{13-48}$ A total of 14,992 patients were analyzed, with a mean age of $48.57 \pm 6.13$. Studies were conducted in various countries in the world (Asia 16, Europe 8, America 9, two or more continents 2, and Australia 1). The median co-morbidity rates of diabetes, hypertension, and dyslipidemia were $36.2 \%$, 43.5\%, and $54.3 \%$, respectively. Median aspartate aminotransferase and alanine aminotransferase levels were $43 \mathrm{U} / \mathrm{L}$ and $62 \mathrm{U} / \mathrm{L}$, respectively.

\section{FIB-4 for predicting AF ( $\geq$ F3)}

Analyzing the diagnostic accuracy of FIB-4 for predicting AF involved 13,764 patients from 32 studies (Table 2 ). As a lower cutoff value for predicting AF, a value from 1.02 to 1.45 was most frequently used (20 studies), and as a higher cutoff value, 2.67 was predominantly used (18 stud-

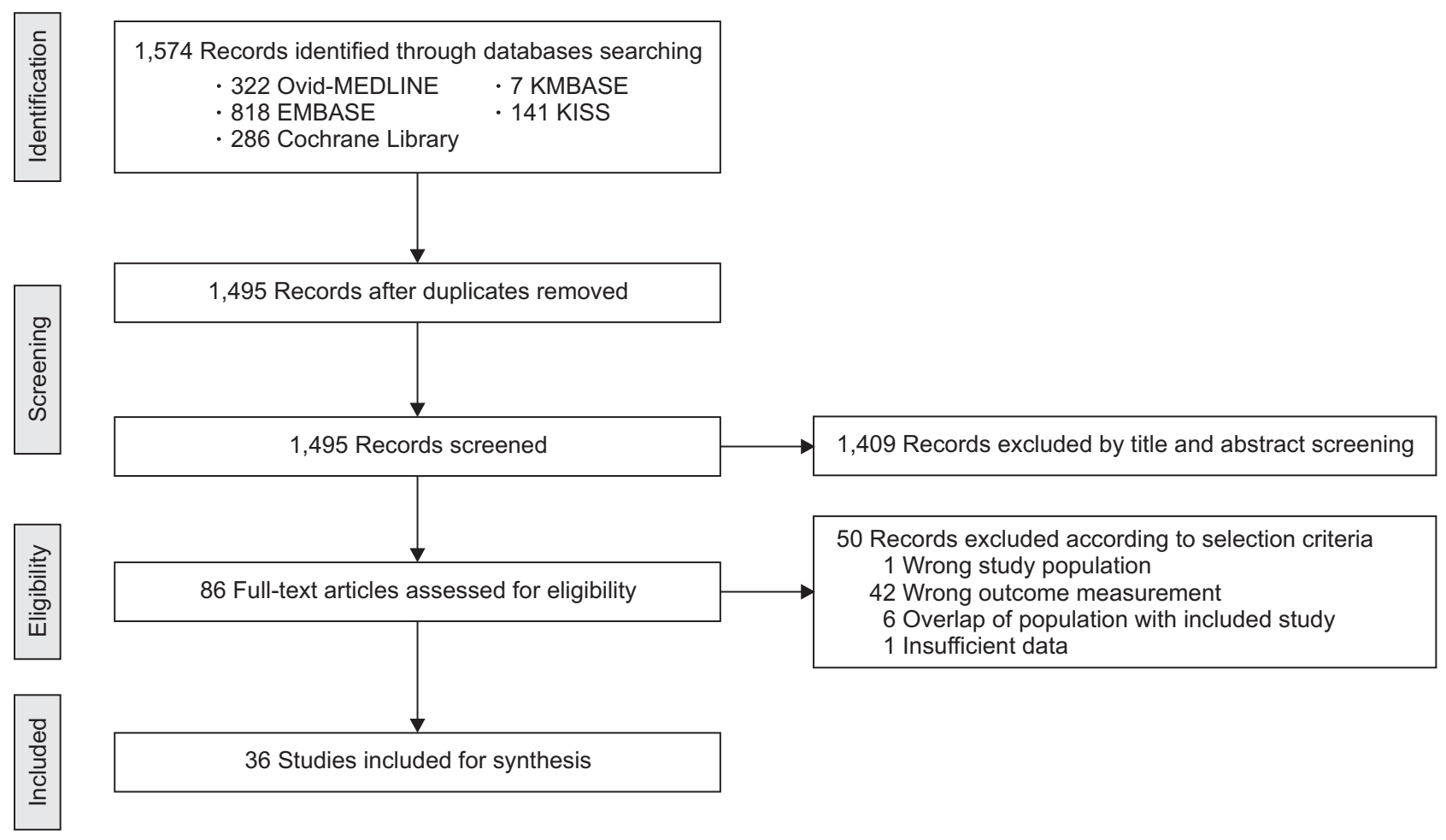

Fig. 1. Flowchart showing the process of study inclusion and exclusion in the systematic review. KMBASE, Korean Medical Database; KISS, Korean Studies Information Service System. 
Table 1. Characteristics and Results of the Included Studies

\begin{tabular}{|c|c|c|c|c|c|c|c|c|c|c|c|c|}
\hline \multirow[b]{2}{*}{ Author (year) } & \multirow[b]{2}{*}{ Location } & \multirow[b]{2}{*}{ Method } & \multirow[b]{2}{*}{$\begin{array}{c}\text { No. of } \\
\text { samples }\end{array}$} & \multirow[b]{2}{*}{$\begin{array}{l}\text { Mean } \\
\text { age, yr }\end{array}$} & \multirow[b]{2}{*}{ Male, \% } & \multicolumn{7}{|c|}{ Clinical characteristics } \\
\hline & & & & & & $\mathrm{DM}, \%$ & HTN, \% & $\begin{array}{c}\text { Dyslip- } \\
\text { idemia, } \\
\%\end{array}$ & $\begin{array}{l}\text { Mean } \\
\text { BMl, } \\
\mathrm{kg} / \mathrm{m}^{2}\end{array}$ & $\begin{array}{c}\text { Mean } \\
\text { waist, } \\
\mathrm{cm}\end{array}$ & $\begin{array}{l}\text { Mean } \\
\text { AST, } \\
\text { U/L }\end{array}$ & $\begin{array}{c}\text { Mean } \\
\text { ALT, } \\
U / L\end{array}$ \\
\hline Aida $(2015)^{13}$ & Japan & FIB-4 & 148 & 61 & 36 & - & - & - & 26.9 & - & 42 & 52 \\
\hline Anstee $(2019)^{14}$ & Global & FIB-4/NFS & 3,123 & 59 & 42 & 68 & - & - & - & - & 44 & 45 \\
\hline Balakrishnan $(2020)^{15}$ & USA & FIB-4/NFS & 99 & 44.7 & 33.9 & 43.5 & - & - & 32.1 & - & 59 & 108 \\
\hline Boursier $(2019)^{16}$ & France & FIB-4/NFS & 938 & 56.5 & 58.5 & 51.1 & - & - & 31.8 & - & 39 & 56 \\
\hline Chan $(2015)^{17}$ & Malaysia & NFS & 147 & 50.5 & 54.4 & 52.4 & 89.1 & - & 29.3 & 98.2 & 41 & 71 \\
\hline Chan $(2019)^{18}$ & Asia & FIB-4/NFS & 583 & 50.9 & 52.9 & 52.3 & 55.1 & 74.5 & 28.9 & 96.5 & 38 & 63 \\
\hline Cui $(2015)^{19}$ & USA & FIB-4 & 102 & 51 & 41.2 & 25.5 & - & - & 31.7 & - & 42.3 & 58 \\
\hline Demir $(2013)^{20}$ & Germany & NFS & 120 & 43.8 & 47.2 & 19.9 & 41.9 & - & 37 & - & 36.8 & 56.6 \\
\hline de Carli $(2019)^{21}$ & Brazil & NFS & 266 & 36.5 & 20.4 & 10.5 & - & - & 44.2 & 123.9 & 24.9 & 32.3 \\
\hline \multirow[t]{2}{*}{ Goh $(2015)^{22}$} & USA & NFS & 238 & 52 & 29.3 & 100 & - & 42 & 37.1 & - & 53.5 & 64 \\
\hline & USA & NFS & 263 & 46 & 46 & 0 & - & 15.2 & 35.2 & - & 58.5 & 78 \\
\hline Joo $(2017)^{23}$ & Korea & FIB-4/NFS & 315 & 55 & 50.8 & 37.8 & 38.4 & - & 27 & 94.6 & 36 & 43 \\
\hline Jun $(2017)^{24}$ & Korea & FIB-4/NFS & 328 & 36.4 & 70.7 & 33 & 14.6 & - & 28.6 & 96.4 & 91.3 & 98.5 \\
\hline Kakisaka $(2018)^{25}$ & Japan & FIB-4 & 63 & 54.9 & 58 & - & - & - & 28.1 & - & 62 & 94 \\
\hline Kao $(2020)^{26}$ & Taiwan & FIB-4 & 73 & 35.3 & 31.5 & 16.9 & 26.8 & - & 41 & 118.3 & 38.2 & 55 \\
\hline Kaya $(2020)^{27}$ & Turkey & FIB-4/NFS & 463 & 46 & 47.5 & 37.8 & 34.8 & - & 31.7 & 104 & 42 & 66 \\
\hline $\operatorname{Kim}(2013)^{28}$ & USA & FIB-4/NFS & 142 & 52.8 & 26.8 & 27.5 & 45.1 & - & 36.32 & - & 47.2 & 60.4 \\
\hline Labenz $(2018)^{29}$ & Germany & FIB-4/NFS & 261 & 51 & 52.5 & 29.9 & - & 37.5 & 30.9 & - & 48 & 60 \\
\hline Lang $(2020)^{30}$ & Germany & FIB-4/NFS & 95 & 50 & 46.2 & 10.8 & 56.9 & - & 30 & 105 & 32.5 & 50.5 \\
\hline $\operatorname{Lum}(2020)^{31}$ & Singapore & FIB-4/NFS & 263 & 50.4 & 52.5 & 49 & - & 66.5 & 30.4 & 113.2 & - & - \\
\hline \multirow[t]{2}{*}{ McPherson $(2013)^{32}$} & UK & FIB-4/NFS & 70 & 54 & 56 & 43 & - & - & 32.9 & 105 & 28 & 28 \\
\hline & UK & FIB-4/NFS & 235 & 48 & 63 & 40 & - & - & 34.4 & 110 & 59 & 95 \\
\hline Meneses $(2020)^{33}$ & Spain & FIB-4/NFS & 50 & 49 & 30 & 26 & 52 & 28 & 44.3 & 135 & 21 & 25 \\
\hline $\operatorname{Nasr}(2016)^{34}$ & Sweden & FIB-4/NFS & 58 & 60.4 & 71 & 53 & 93 & - & 28 & 102 & 34 & 60 \\
\hline \multirow[t]{2}{*}{ Ooi $(2017)^{35}$} & Australia & FIB-4/NFS & 101 & 49 & 33.7 & 34.7 & 79.2 & 73.3 & 41.9 & - & - & - \\
\hline & Australia & FIB-4/NFS & 53 & 43 & 30.2 & 24.5 & 45.3 & 19.2 & 46.6 & - & - & - \\
\hline \multirow[t]{2}{*}{ Patel $(2018)^{36}$} & USA & FIB-4/NFS & 114 & 41.8 & 79 & 30 & - & - & 33.9 & - & 49 & 84 \\
\hline & USA & FIB-4/NFS & 151 & 60 & 95 & 70 & - & - & 33.7 & - & 48.2 & 54.6 \\
\hline $\begin{array}{l}\text { Pérez-Gutiérrez } \\
(2013)^{37}\end{array}$ & Mexico & FIB-4/NFS & 243 & 48.6 & 49 & 21.5 & - & - & - & - & 57.6 & 73 \\
\hline \multirow[t]{2}{*}{ Petta $(2015)^{38}$} & Italy & FIB-4/NFS & 179 & 45.4 & 67.5 & 19.5 & 24 & - & 29.3 & - & 45.7 & 80.3 \\
\hline & Italy & FIB-4/NFS & 142 & 43.9 & 71.8 & 15.4 & 11.9 & - & 27.4 & - & 42.2 & 75.6 \\
\hline Petta $(2019)^{39}$ & Global & FIB-4/NFS & 968 & 50.1 & 62.9 & 37 & 39.4 & - & 29.3 & - & 46.1 & 76.1 \\
\hline Siddiqui $(2020)^{40}$ & USA & FIB-4/NFS & 1,904 & 50.3 & 37 & 39 & 58 & 62 & 34.4 & - & 51.2 & 69.8 \\
\hline Singh $(2020)^{41}$ & USA & FIB-4/NFS & 1,134 & 51.1 & 35.4 & 100 & 74.5 & 70.8 & 35.5 & - & 27 & 28 \\
\hline $\begin{array}{l}\text { Treeprasertsuk } \\
(2016)^{42}\end{array}$ & Thailand & FIB-4/NFS & 139 & 40.9 & 47 & 38 & - & - & 36.1 & - & 38 & 56 \\
\hline Wong $(2008)^{43}$ & Hong Kong & NFS & 128 & 46 & 59 & 57 & 48 & - & 28.5 & 95 & 43 & 75 \\
\hline Wong $(2010)^{44}$ & Hong Kong & FIB-4/NFS & 246 & 51 & 54.9 & 36.2 & 40.2 & - & 28 & 94 & - & 75 \\
\hline Xun $(2012)^{45}$ & China & FIB-4/NFS & 152 & 37.1 & 79.6 & 32.2 & - & - & 26.1 & - & 61 & 100 \\
\hline Yang $(2019)^{46}$ & China & FIB-4/NFS & 453 & 36.56 & 58.9 & 30.2 & 34.8 & - & 26.93 & - & 74.12 & 135.11 \\
\hline Yoneda $(2013)^{47}$ & Japan & FIB-4/NFS & 235 & 59.9 & - & 46 & - & 63.8 & 26.9 & - & 24.7 & 23.7 \\
\hline Zhou $(2019)^{48}$ & China & FIB-4/NFS & 207 & 41.8 & 73 & 24.6 & 20.3 & 46.6 & 27 & 91.2 & 45.7 & 49 \\
\hline
\end{tabular}

DM, diabetes mellitus; HTN, hypertension; BMI, body mass index; AST, aspartate aminotransferase; ALT, alanine aminotransferase; FIB-4, Fibrosis-4; NFS, nonalcoholic fatty liver disease (NAFLD) fibrosis score.

ies). Regarding the FIB-4 index, pooled sensitivity was 0.42 ( $95 \%$ confidence interval [CI], 0.33 to 0.51 ) and pooled specificity was 0.93 ( $95 \%$ CI, 0.91 to 0.95$)$. Pooled diagnostic odds ratio (DOR) with $95 \%$ CI was 10.83 (7.55 to 15.54 ) with $\mathrm{I}^{2}$ of $85 \%(\mathrm{p}<0.01)$. Summary statistics of FIB-4 at various thresholds for prediction of $\mathrm{AF}$ and forest plots are presented in Supplementary Table 1 and Supplementary
Fig. 1. The area under the receiver operating characteristic curve (AUC) of summary receiver operating characteristic (SROC) was 0.76 (95\% CI, 0.74 to 0.81 ) (Fig. 2A).

\section{NFS for predicting AF ( $\geq F 3)$}

Analyzing the diagnostic accuracy of NFS for predicting AF involved 13,337 patients from 33 studies (Table 2). As 


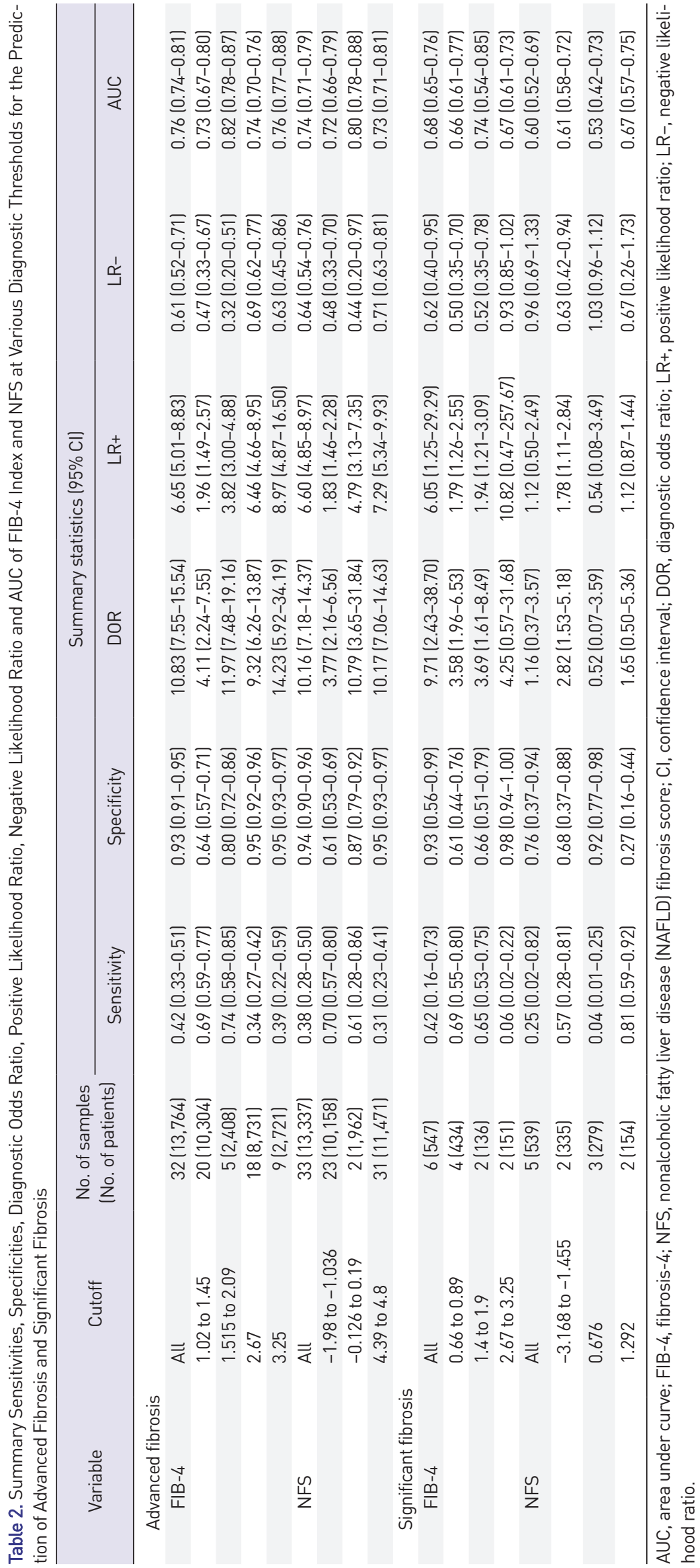



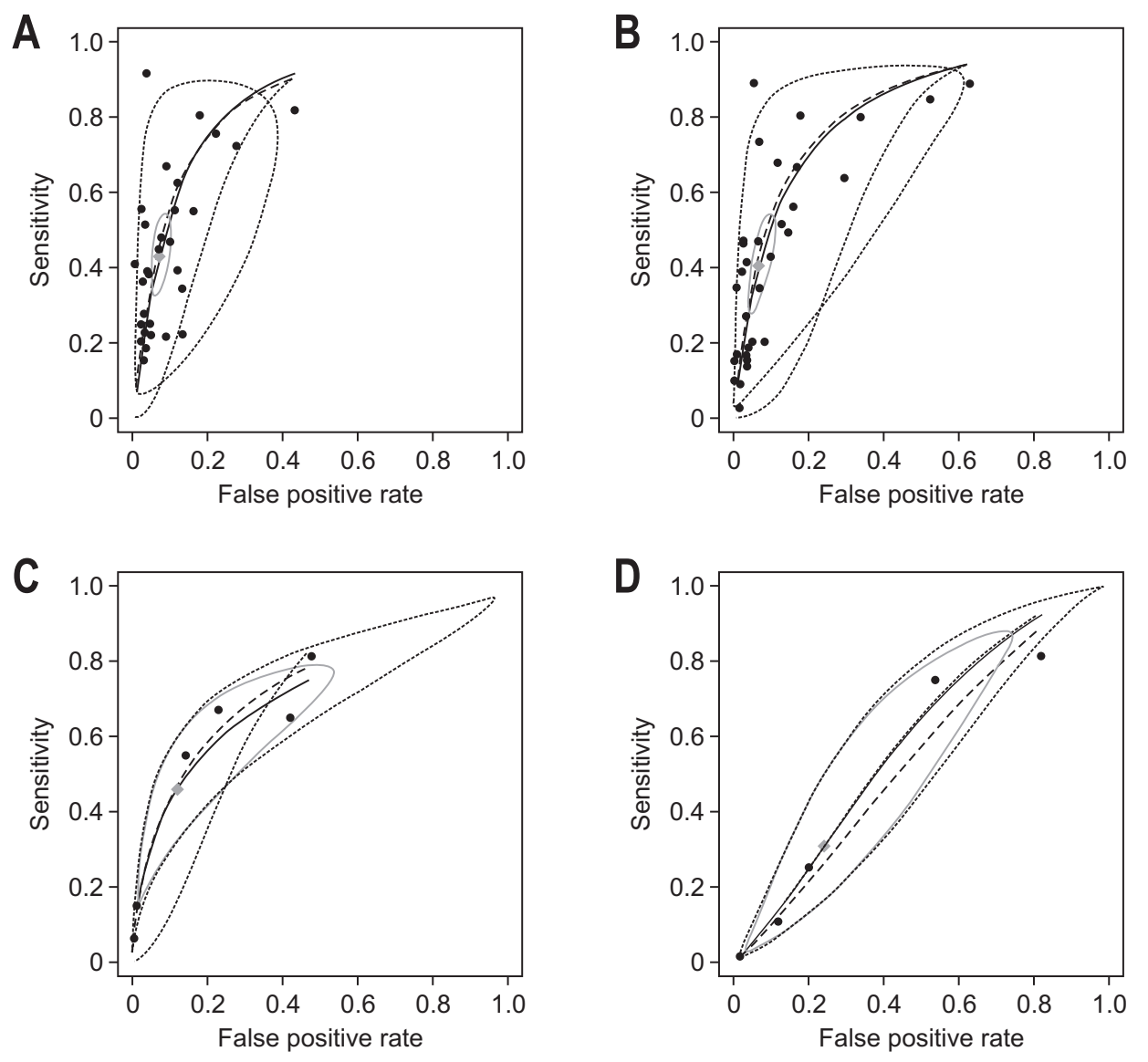

Fig. 2. The summary receiver operating characteristic curve. (A) FIB4 index for predicting advanced fibrosis ( $\geq F 3)$, (B) NFS for predicting advanced fibrosis ( $\geq F 3$ ), (C) FIB-4 index for predicting significant fibrosis ( $\geq F 2)$, and (D) NFS for predicting significant fibrosis $(\geq F 2)$ in patients with NAFLD.

FIB-4, fibrosis-4; NFS, nonalcoholic fatty liver disease (NAFLD) fibrosis score.

a lower cutoff value for predicting AF, a value from -1.98 to -1.03 was most frequently used ( 23 studies), and as a higher cutoff value, 4.39 to 4.8 was predominantly used (31 studies). For NFS, pooled sensitivity was 0.38 ( $95 \%$ CI, 0.28 to 0.50 ) and pooled specificity was 0.94 ( $95 \% \mathrm{CI}, 0.90$ to 0.96 ) (Table 2). Pooled DOR with $95 \%$ CI was 10.16 (7.18 to 14.37$)$ with $\mathrm{I}^{2}$ of $85 \%(\mathrm{p}<0.01)$, indicating heterogeneity of the meta-analysis (Table 2, Supplementary Fig. 2). Summary statistics of FIB-4 at various thresholds for prediction of AF and forest plots are presented in Supplementary Table 2 and Supplementary Fig. 2. The AUC of SROC was 0.74 ( $95 \%$ CI, 0.71 to 0.79 ) (Fig. $2 \mathrm{~B}$ ).

\section{FIB-4 and NFS for predicting SF ( $\geq F 2)$}

Studies on SF were relatively scarce, compared to studies on AF (32 studies vs 6 studies) (Table 2). Also, while the cutoff for AF was consistent for each study, the cutoff for SF differed significantly between studies. In regard to the FIB-4 index, the pooled sensitivity was 0.42 (95\% CI, 0.16 to 0.73 ) and pooled specificity was 0.93 (95\% CI, 0.56 to 0.99 ). The pooled DOR with $95 \%$ CI was 9.71 (2.43 to $38.70)$ with $\mathrm{I}^{2}$ of $0 \%(\mathrm{p}=0.54)$. The AUC of SROC was 0.68 ( $95 \%$ CI, 0.65 to 0.76 ) (Fig. 2C). Summary statistics of FIB4 at various thresholds for prediction of SF and forest plots are presented in Supplementary Table 3 and Supplementary Fig. 3.

In regard to NFS, the pooled sensitivity was 0.25 (95\% CI, 0.02 to 0.82$)$ and pooled specificity was $0.76(95 \% \mathrm{CI}$, 0.37 to 0.94$)$. The pooled DOR with $95 \%$ CI was 1.16 (0.37 to 3.57$)$ with $\mathrm{I}^{2}$ of $0 \%(\mathrm{p}=0.54)$, indicating homogeneity. The AUC of SROC was 0.60 (95\% CI, 0.52 to 0.69 ) (Fig. 2D). Summary statistics of NFS at various thresholds for prediction of SF and forest plots are presented in Supplementary Table 4 and Supplementary Fig. 4.

\section{Difference in diagnosis accuracy according to region and body mass index}

Finally, we analyzed whether the accuracy of NFS or FIB-4 differs according to the study area and body mass index (BMI) (Table 3). Regions could be classified into three categories, Asia/Europe/America, except for the two studies that were conducted globally on two or more continents. We found that the accuracy of FIB-4 or NFS to predict F3 relatively increased in Europe (FIB-4: pooled DOR 16.37, NFS: pooled DOR 21.94) compared with Asia (FIB4: pooled DOR 6.09, NFS: pooled DOR 6.22) or America (FIB-4: pooled DOR 6.23, NFS: pooled DOR 3.70). For $\mathrm{BMI}$, individual patient BMI data could not be obtained, 


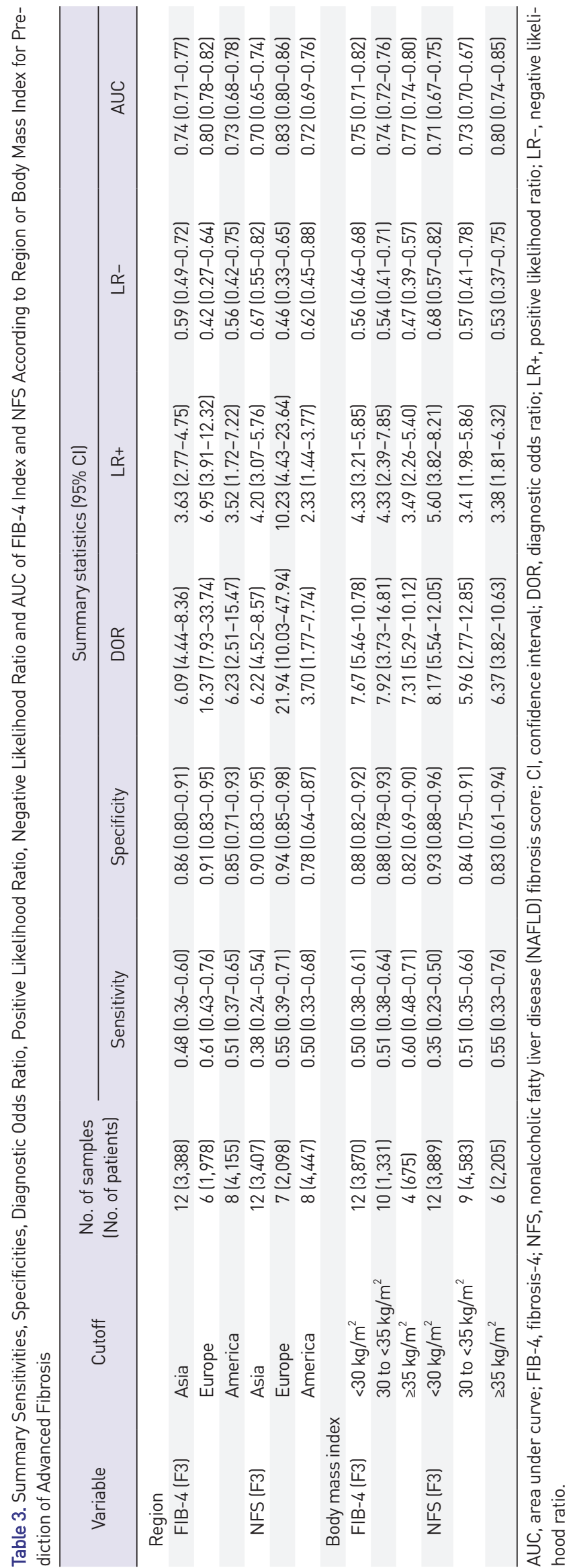


so the average BMI for each study was used. In all studies, BMI was $25 \mathrm{~kg} / \mathrm{m}^{2}$ or higher (minimum, $26.1 \mathrm{~kg} / \mathrm{m}^{2}$ ), and BMI was classified into three groups: $<30 \mathrm{~kg} / \mathrm{m}^{2}, 30$ to $<35$ $\mathrm{kg} / \mathrm{m}^{2}$, and $35 \mathrm{~kg} / \mathrm{m}^{2}$ or higher. As a result of stratification analysis, BMI values did not significantly affect the accuracy of NFS or FIB-4. Meta-regression analysis was added for the effect of BMI on DOR of FIB-4 or NFS, but it was not significant as well (Table 4).

\section{DISCUSSION}

This systematic review and meta-analysis of 36 relevant studies indicated that the FIB-4 index and NFS can be effectively used to predict the degree of liver fibrosis in NAFLD. Additionally, our results demonstrated that the diagnostic accuracy of FIB-4 and NFS is relatively higher in predicting AF than in SF. Our study holds significance in its ability to assist clinicians in deciding treatments for NAFLD patients, by accurately predicting the degree of liver fibrosis.

Out of all panels based on serological markers, the NFS system was the most frequently studied. NFS is a scoring system based on 733 NAFLD patients diagnosed by liver biopsy. ${ }^{10}$ In previous studies, the diagnostic ability of NFS for hepatic fibrosis was AUC 0.82-0.88. Given such precedent, two cutoff values $(<-1.455$ [low probability, negative predictive value $88 \%$ to $93 \%$ ] and $>0.676$ [high probability, positive predictive value $82 \%$ to $90 \%$ ]) were proposed. ${ }^{10}$ Existing meta-studies on the diagnostic predictive ability of NFS in NAFLD have reported up to AUC 0.73-0.86, which is consistent with the results of our study. ${ }^{49-51}$ However, in the NFS scoring system, there are cases in which either high or low probability for advanced liver fibrosis cannot be classified (indeterminate probability); in such cases, a liver biopsy may be necessary. ${ }^{52}$
On the other hand, the FIB-4 index was created by Sterling et al. ${ }^{9}$ using a cohort of 832 human immunodeficiency virus/hepatitis $\mathrm{C}$ virus co-infected patients. Compared to NFS, FIB-4 has been widely validated for liver diseases of relatively diverse etiologies. ${ }^{53-55}$ In previous meta-studies, the diagnostic AUC of FIB-4 for AF in NAFLD was 0.76. Like NFS, FIB- 4 has two cutoffs: FIB- $4<1.30$ can be interpreted as low possibility of AF, while FIB-4 $>2.67$ can be interpreted as high possibility of AF. In addition to NFS and FIB-4, other noninvasive markers exist.

However, in regard to NAFLDs, NFS and FIB-4 showed higher diagnostic abilities than other noninvasive panels have. Notably, both markers demonstrated a diagnostic ability of AF similar to that of magnetic resonance elastography. ${ }^{56}$ Therefore, most of the NAFLD guidelines recommend NFS and FIB-4 as screening tools for diagnosing $\mathrm{AF}^{57-59}$

First, our study found that FIB-4 has better diagnostic performance than NFS in predicting AF (AUC 0.76 vs AUC 0.74). Similar results have been manifested in previous studies. According to a meta-analysis conducted on 13,046 NAFLD subjects in 2017, AUROC of FIB-4 and NFS for the prediction of AF were 0.80 and 0.78 , respectively, indicating that FIB-4 index has higher diagnostic accuracy than NFS. ${ }^{49}$ Also, in a meta-analysis of 5,735 NAFLD patients in 2021, AUROC of FIB-4 index and NFS were 0.76 (95\% CI, 0.74 to 0.77$)$ and 0.73 (95\% CI, 0.71 to $0.75)$, respectively. ${ }^{60}$

Secondly, according to our data, the ability to predict SF was inferior to the AF predictive abilities of both markers. We can assume diagnostic accuracy of each scoring system is higher for more severe types of patients, as supported by previous studies. According to a study by Xiao et al., ${ }^{49} \mathrm{AU}$ ROC values of FIB-4 and NFS increased according to SF, $\mathrm{AF}$, and liver cirrhosis $(0.75,0.80$ and 0.85 for FIB- 4 and $0.72,0.78$ and 0.83 for NFS).

Table 4. Meta-Regression for Diagnostic Odds Ratio of Each Measurement

\begin{tabular}{|c|c|c|c|c|}
\hline \multirow{2}{*}{ Variable } & \multicolumn{2}{|l|}{ FIB-4 for AF } & \multicolumn{2}{|c|}{ NFS for AF } \\
\hline & Coefficient (95\% CI) & $\mathrm{p}$-value & Coefficient $(95 \%$ CI) & $p$-value \\
\hline Mean age, yr & 0.063 (0.022 to 0.104$)$ & 0.002 & 0.095 (0.048 to 0.143 ) & $<0.001$ \\
\hline Proportion of male, \% & $-0.019(-0.037$ to -0.001$)$ & 0.034 & $-0.022(-0.043$ to -0.001$)$ & 0.040 \\
\hline $\mathrm{DM}, \%$ & $0.005(-0.011$ to 0.021$)$ & 0.561 & $0.013(-0.001$ to 0.028$)$ & 0.065 \\
\hline HTN, \% & $0.028(-0.001$ to 0.045$)$ & 0.055 & $0.021(-0.003$ to 0.046$)$ & 0.089 \\
\hline Dyslipidemia, \% & $-0.009(-0.067$ to 0.048$)$ & 0.747 & $0.005(-0.028$ to 0.039$)$ & 0.758 \\
\hline Mean BMI, kg/m² & 0.004 (-0.086 to 0.095$)$ & 0.919 & $0.062(-0.032$ to 0.156$)$ & 0.196 \\
\hline Mean waist, cm & $-0.009(-0.075$ to 0.056$)$ & 0.774 & $0.001(-0.070$ to 0.071$)$ & 0.997 \\
\hline Mean AST & $-0.020(-0.041$ to 0.001$)$ & 0.060 & $-0.023(-0.047$ to 0.001$)$ & 0.055 \\
\hline Mean ALT & $-0.014(-0.026$ to -0.002$)$ & 0.017 & $-0.018(-0.032$ to -0.005$)$ & 0.007 \\
\hline
\end{tabular}

FIB-4, fibrosis-4; AF, advanced fibrosis; NFS, nonalcoholic fatty liver disease (NAFLD) fibrosis score; Cl, confidence interval; DM, diabetes mellitus; HTN, hypertension; BMI, body mass index; AST, aspartate aminotransferase; ALT, alanine aminotransferase. 
Third finding of our study is that the ability of FIB-4 or NFS to predict AF is lower than that of previous reports. This is interpreted because of the diversity of the patient population used in our study analysis. In fact, when comparing existing studies or meta-analyses, the AUC of FIB4 or NFS gradually decreases as the number of patients or studies analyzed in the study increases. When analyzing 145 patients, AUC of FIB-4 and NFS was 0.86 and 0.81 , respectively. ${ }^{61}$ In 1,038 patients, AUC of FIB-4 was $0.849 .{ }^{50}$ When analyzed in 5,735 patients from 37 studies, the AUC for FIB-4 and NFS was 0.76 and 0.73 , which is almost consistent with our study results. ${ }^{60}$ In our study, the AUCs of FIB- 4 and NFS were 0.76 and 0.74 , respectively.

Finally, our study found that accuracy of FIB-4 or NFS to predict F3 relatively increased in Europe compared with Asia or America. This is interpreted because the proportion of Caucasians in the cohort study in which FIB or NFS was developed was relatively high, 79\% for FIB-4 and 90\% for NFS. ${ }^{9,10,62}$ Therefore, the accuracy of FIB or NFS is relatively low in Asian countries or in the multiracial America continent.

Advantages of these markers in NAFLD include low cost, quick diagnosis, and easy repeatability. In primary care, the use of noninvasive markers can increase early detection of AF, decrease avoidable referral of patients with mild diseases and ensure cost-effectiveness. ${ }^{63}$ Therefore, many experts recommend implementing a two-tier approach to improve resource utilization. ${ }^{18,57,64}$ On the other hand, compared to transient elastography or magnetic resonance elastography, disadvantages such as the low ability to diagnose AF should always be noted. ${ }^{60}$

The strength of our meta-analysis is its focus on comparing the diagnostic accuracies of two noninvasive and routinely usable scoring systems to predict the degree of liver fibrosis in NAFLD patients. To the best of our knowledge, this meta-analysis has the largest sample size, amongst those that compare the diagnostic accuracy of noninvasive scoring systems for AF and SF in NAFLD patients. However, there are some limitations to consider. First, the biggest limitation of this study is that it lacks new information compared to the existing meta-analysis related to FIB-4 or NFS. Before starting the study, we reviewed the existing meta-analysis literature and found that more literature than expected was not included in the analysis. Therefore, in order to obtain more accurate results, it was determined that accurate inclusion criteria should be reapplied, and as a result, a wider range of papers could be analyzed than existing meta-analysis papers. Eventually, the results of our study are not very different from the existing meta-analyses, and thus provide few new revelations. However, our study has clinical significance as the most extensive analytical research on this subject. Second, limiting our review to manuscripts published only in English may have caused publication bias. We conducted a funnel plot and Egger's test, later to confirm that our study has no publication bias with $\mathrm{p}=0.135$ in FIB- 4 for AF. However, there was a publication bias in FIB-4 for SF, and NSF for AF and SF with $\mathrm{p}<0.05$, which may limit the credibility of our results. Third, only two noninvasive scoring systems were the focus of our analysis. Other noninvasive scoring systems such as BMI, aspartate aminotransferase/alanine aminotransferase ratio, diabetes (BARD) score, and aspartate aminotransferase to platelet ratio tests were not considered. ${ }^{50,65}$ Fourth, our research focused on liver fibrosis and did not consider the degree of hepatic steatosis. Furthermore, the included studies did not provide sufficient information on the patients' duration of NAFLD or past treatments, which can affect the incidence and severity of liver fibrosis.

In summary, both FIB-4 index and NFS were useful in predicting the degree of liver fibrosis in NAFLD. The diagnostic accuracy of these scoring systems was higher in predicting AF than in SF. Thus, the FIB-4 index and NFS may be considered as alternative diagnostic methods to liver biopsy when predicting the level of fibrosis in NAFLD.

\section{CONFLICTS OF INTEREST}

No potential conflict of interest relevant to this article was reported.

\section{ACKNOWLEDGEMENTS}

This research was supported by Basic Science Research Program through the National Research Foundation of Korea (NRF) funded by the Ministry of Education, Science and Technology (2021R1G1A1007886), and in part by the Soonchunhyang University Research Fund.

\section{AUTHOR CONTRIBUTIONS}

Study concept and design: D.W.J. Provision of study materials or patients: M.C., H.W.L. Collection and assembly of data: S.H.K., Y.C., S.B.A., D.S.S. Data analysis and interpretation: B.L., S.H., J.J.Y. Manuscript writing: S.H., J.L., J.J.Y. Final approval of manuscript: all authors. 


\section{ORCID}

Sangsoo Han

Miyoung Choi

Bora Lee

Hye-Won Lee

Seong Hee Kang

Yuri Cho

Sang Bong Ahn

Do Seon Song

Dae Won Jun

Jieun Lee

Jeong-Ju Yoo https://orcid.org/0000-0002-9709-3332

https://orcid.org/0000-0002-2424-9965

https://orcid.org/0000-0002-6322-5712

https://orcid.org/0000-0002-3552-3560

https://orcid.org/0000-0002-0783-509X

https://orcid.org/0000-0002-4488-5352

https://orcid.org/0000-0001-7419-5259

https://orcid.org/0000-0002-8278-4878

https://orcid.org/0000-0002-2875-6139

https://orcid.org/0000-0001-9494-2493

https://orcid.org/0000-0002-7802-0381

\section{SUPPLEMENTARY MATERIALS}

Supplementary materials can be accessed at https://doi. org/10.5009/gnl210391.

\section{REFERENCES}

1. Rinella ME. Nonalcoholic fatty liver disease: a systematic review. JAMA 2015;313:2263-2273.

2. Younossi ZM, Koenig AB, Abdelatif D, Fazel Y, Henry L, Wymer M. Global epidemiology of nonalcoholic fatty liver disease: meta-analytic assessment of prevalence, incidence, and outcomes. Hepatology 2016;64:73-84.

3. Williams CD, Stengel J, Asike MI, et al. Prevalence of nonalcoholic fatty liver disease and nonalcoholic steatohepatitis among a largely middle-aged population utilizing ultrasound and liver biopsy: a prospective study. Gastroenterology 2011;140:124-131.

4. Sesti G, Sciacqua A, Fiorentino TV, Perticone M, Succurro E, Perticone F. Association between noninvasive fibrosis markers and cardio-vascular organ damage among adults with hepatic steatosis. PLoS One 2014;9:e104941.

5. Charlton M. Nonalcoholic fatty liver disease: a review of current understanding and future impact. Clin Gastroenterol Hepatol 2004;2:1048-1058.

6. Farrell GC, Larter CZ. Nonalcoholic fatty liver disease: from steatosis to cirrhosis. Hepatology 2006;43(2 Suppl 1):S99S112.

7. Nalbantoglu IL, Brunt EM. Role of liver biopsy in nonalcoholic fatty liver disease. World J Gastroenterol 2014;20:90269037.

8. Ratziu V, Charlotte F, Heurtier A, et al. Sampling variability of liver biopsy in nonalcoholic fatty liver disease. Gastroenterology 2005;128:1898-1906.

9. Sterling RK, Lissen E, Clumeck N, et al. Development of a simple noninvasive index to predict significant fibrosis in patients with $\mathrm{HIV} / \mathrm{HCV}$ coinfection. Hepatology 2006;43:1317-1325.

10. Angulo P, Hui JM, Marchesini G, et al. The NAFLD fibrosis score: a noninvasive system that identifies liver fibrosis in patients with NAFLD. Hepatology 2007;45:846-854.

11. Whiting P, Rutjes AW, Reitsma JB, Bossuyt PM, Kleijnen J. The development of QUADAS: a tool for the quality assessment of studies of diagnostic accuracy included in systematic reviews. BMC Med Res Methodol 2003;3:25.

12. Hayden JA, van der Windt DA, Cartwright JL, Côté P, Bombardier C. Assessing bias in studies of prognostic factors. Ann Intern Med 2013;158:280-286.

13. Aida Y, Abe H, Tomita Y, et al. Serum immunoreactive collagen IV detected by monoclonal antibodies as a marker of severe fibrosis in patients with non-alcoholic fatty liver disease. J Gastrointestin Liver Dis 2015;24:61-68.

14. Anstee QM, Lawitz EJ, Alkhouri N, et al. Noninvasive tests accurately identify advanced fibrosis due to NASH: baseline data from the STELLAR trials. Hepatology 2019;70:15211530 .

15. Balakrishnan M, Seth A, Cortes-Santiago N, et al. External validation of four point-of-care noninvasive scores for predicting advanced hepatic fibrosis in a predominantly Hispanic NAFLD population. Dig Dis Sci 2021;66:2387-2393.

16. Boursier J, Guillaume M, Leroy V, et al. New sequential combinations of non-invasive fibrosis tests provide an accurate diagnosis of advanced fibrosis in NAFLD. J Hepatol 2019;71:389-396.

17. Chan WK, Nik Mustapha NR, Mahadeva S. A novel 2-step approach combining the NAFLD fibrosis score and liver stiffness measurement for predicting advanced fibrosis. Hepatol Int 2015;9:594-602.

18. Chan WK, Treeprasertsuk S, Goh GB, et al. Optimizing use of nonalcoholic fatty liver disease fibrosis score, fibrosis-4 score, and liver stiffness measurement to identify patients with advanced fibrosis. Clin Gastroenterol Hepatol 2019;17:2570-2580.

19. Cui J, Ang B, Haufe W, et al. Comparative diagnostic accuracy of magnetic resonance elastography vs. eight clinical prediction rules for non-invasive diagnosis of advanced fibrosis in biopsy-proven non-alcoholic fatty liver disease: a prospective study. Aliment Pharmacol Ther 2015;41:12711280.

20. Demir M, Lang S, Schlattjan M, et al. NIKEI: a new inexpensive and non-invasive scoring system to exclude advanced fibrosis in patients with NAFLD. PLoS One 2013;8:e58360.

21. de Carli MA, de Carli LA, Correa MB, Junqueira G Jr, Tovo CV, Coral GP. Performance of noninvasive scores for the diagnosis of advanced liver fibrosis in morbidly obese with nonalcoholic fatty liver disease. Eur J Gastroenterol Hepatol 
2020;32:420-425.

22. Goh GB, Pagadala MR, Dasarathy J, et al. Clinical spectrum of non-alcoholic fatty liver disease in diabetic and nondiabetic patients. BBA Clin 2014;3:141-145.

23. Joo SK, Kim W, Kim D, et al. Steatosis severity affects the diagnostic performances of noninvasive fibrosis tests in nonalcoholic fatty liver disease. Liver Int 2018;38:331-341.

24. Jun DW, Kim SG, Park SH, et al. External validation of the non-alcoholic fatty liver disease fibrosis score for assessing advanced fibrosis in Korean patients. J Gastroenterol Hepatol 2017;32:1094-1099.

25. Kakisaka K, Suzuki Y, Fujiwara Y, et al. Evaluation of ballooned hepatocytes as a risk factor for future progression of fibrosis in patients with non-alcoholic fatty liver disease. J Gastroenterol 2018;53:1285-1291.

26. Kao WY, Chang IW, Chen CL, et al. Fibroscan-based score to predict significant liver fibrosis in morbidly obese patients with nonalcoholic fatty liver disease. Obes Surg 2020;30:1249-1257.

27. Kaya E, Bakir A, Kani HT, Demirtas CO, Keklikkiran C, Yilmaz Y. Simple noninvasive scores are clinically useful to exclude, not predict, advanced fibrosis: a study in Turkish patients with biopsy-proven nonalcoholic fatty liver disease. Gut Liver 2020;14:486-491.

28. Kim D, Kim WR, Talwalkar JA, Kim HJ, Ehman RL. Advanced fibrosis in nonalcoholic fatty liver disease: noninvasive assessment with MR elastography. Radiology 2013;268:411-419.

29. Labenz C, Huber Y, Kalliga E, et al. Predictors of advanced fibrosis in non-cirrhotic non-alcoholic fatty liver disease in Germany. Aliment Pharmacol Ther 2018;48:1109-1116.

30. Lang S, Farowski F, Martin A, et al. Prediction of advanced fibrosis in non-alcoholic fatty liver disease using gut microbiota-based approaches compared with simple non-invasive tools. Sci Rep 2020;10:9385.

31. Lum J, Cheah M, Leow WQ, et al. Clinical profile of nonalcoholic fatty liver disease in nonobese patients. J Gastroenterol Hepatol 2021;36:257-261.

32. McPherson S, Anstee QM, Henderson E, Day CP, Burt AD. Are simple noninvasive scoring systems for fibrosis reliable in patients with NAFLD and normal ALT levels? Eur J Gastroenterol Hepatol 2013;25:652-658.

33. Meneses D, Olveira A, Corripio R, et al. Performance of noninvasive liver fibrosis scores in the morbid obese patient, same scores but different thresholds. Obes Surg 2020;30:2538-2546.

34. Nasr P, Hilliges A, Thorelius L, Kechagias S, Ekstedt M. Contrast-enhanced ultrasonography could be a non-invasive method for differentiating none or mild from severe fibrosis in patients with biopsy proven non-alcoholic fatty liver disease. Scand J Gastroenterol 2016;51:1126-1132.
35. Ooi GJ, Burton PR, Doyle L, et al. Modified thresholds for fibrosis risk scores in nonalcoholic fatty liver disease are necessary in the obese. Obes Surg 2017;27:115-125.

36. Patel YA, Gifford EJ, Glass LM, et al. Identifying nonalcoholic fatty liver disease advanced fibrosis in the Veterans Health Administration. Dig Dis Sci 2018;63:2259-2266.

37. Pérez-Gutiérrez OZ, Hernández-Rocha C, Candia-Balboa RA, et al. Validation study of systems for noninvasive diagnosis of fibrosis in nonalcoholic fatty liver disease in Latin population. Ann Hepatol 2013;12:416-424.

38. Petta S, Vanni E, Bugianesi E, et al. The combination of liver stiffness measurement and NAFLD fibrosis score improves the noninvasive diagnostic accuracy for severe liver fibrosis in patients with nonalcoholic fatty liver disease. Liver Int 2015;35:1566-1573.

39. Petta S, Wai-Sun Wong V, Bugianesi E, et al. Impact of obesity and alanine aminotransferase levels on the diagnostic accuracy for advanced liver fibrosis of noninvasive tools in patients with nonalcoholic fatty liver disease. Am J Gastroenterol 2019;114:916-928.

40. Siddiqui MS, Yamada G, Vuppalanchi R, et al. Diagnostic accuracy of noninvasive fibrosis models to detect change in fibrosis stage. Clin Gastroenterol Hepatol 2019;17:18771885.

41. Singh A, Gosai F, Siddiqui MT, et al. Accuracy of noninvasive fibrosis scores to detect advanced fibrosis in patients with type-2 diabetes with biopsy-proven nonalcoholic fatty liver disease. J Clin Gastroenterol 2020;54:891-897.

42. Treeprasertsuk S, Piyachaturawat P, Soontornmanokul T, Wisedopas-Klaikaew N, Komolmit P, Tangkijavanich P. Accuracy of noninvasive scoring systems to assess advanced liver fibrosis in Thai patients with nonalcoholic fatty liver disease. Asian Biomed 2016;10:s49-s55.

43. Wong VW, Wong GL, Chim AM, et al. Validation of the NAFLD fibrosis score in a Chinese population with low prevalence of advanced fibrosis. Am J Gastroenterol 2008;103:1682-1688.

44. Wong VW, Vergniol J, Wong GL, et al. Diagnosis of fibrosis and cirrhosis using liver stiffness measurement in nonalcoholic fatty liver disease. Hepatology 2010;51:454-462.

45. Xun YH, Fan JG, Zang GQ, et al. Suboptimal performance of simple noninvasive tests for advanced fibrosis in Chinese patients with nonalcoholic fatty liver disease. J Dig Dis 2012;13:588-595.

46. Yang M, Jiang L, Wang Y, et al. Step layered combination of noninvasive fibrosis models improves diagnostic accuracy of advanced fibrosis in nonalcoholic fatty liver disease. J Gastrointestin Liver Dis 2019;28:289-296.

47. Yoneda M, Imajo K, Eguchi Y, et al. Noninvasive scoring systems in patients with nonalcoholic fatty liver disease with normal alanine aminotransferase levels. J Gastroenterol 
2013;48:1051-1060.

48. Zhou YJ, Ye FZ, Li YY, et al. Individualized risk prediction of significant fibrosis in non-alcoholic fatty liver disease using a novel nomogram. United European Gastroenterol J 2019;7:1124-1134.

49. Xiao G, Zhu S, Xiao X, Yan L, Yang J, Wu G. Comparison of laboratory tests, ultrasound, or magnetic resonance elastography to detect fibrosis in patients with nonalcoholic fatty liver disease: a meta-analysis. Hepatology 2017;66:14861501.

50. Sun W, Cui H, Li N, et al. Comparison of FIB-4 index, NAFLD fibrosis score and BARD score for prediction of advanced fibrosis in adult patients with non-alcoholic fatty liver disease: a meta-analysis study. Hepatol Res 2016;46:862870 .

51. Jaruvongvanich V, Wijarnpreecha K, Ungprasert P. The utility of NAFLD fibrosis score for prediction of mortality among patients with nonalcoholic fatty liver disease: a systematic review and meta-analysis of cohort study. Clin Res Hepatol Gastroenterol 2017;41:629-634.

52. Bril F, Sninsky JJ, Baca AM, et al. Hepatic steatosis and insulin resistance, but not steatohepatitis, promote atherogenic dyslipidemia in NAFLD. J Clin Endocrinol Metab 2016;101:644-652.

53. Vilar-Gomez E, Chalasani N. Non-invasive assessment of non-alcoholic fatty liver disease: clinical prediction rules and blood-based biomarkers. J Hepatol 2018;68:305-315.

54. Sonneveld MJ, Brouwer WP, Chan HL, et al. Optimisation of the use of APRI and FIB-4 to rule out cirrhosis in patients with chronic hepatitis B: results from the SONIC-B study. Lancet Gastroenterol Hepatol 2019;4:538-544.

55. Vallet-Pichard A, Mallet V, Nalpas B, et al. FIB-4: an inexpensive and accurate marker of fibrosis in HCV infection. comparison with liver biopsy and fibrotest. Hepatology 2007;46:32-36.

56. Imajo K, Kessoku T, Honda Y, et al. Magnetic resonance imaging more accurately classifies steatosis and fibrosis in patients with nonalcoholic fatty liver disease than transient elastography. Gastroenterology 2016;150:626-637.

57. Kang SH, Lee HW, Yoo JJ, et al. KASL clinical practice guide- lines: management of nonalcoholic fatty liver disease. Clin Mol Hepatol 2021;27:363-401.

58. European Association for the Study of the Liver (EASL); European Association for the Study of Diabetes (EASD); European Association for the Study of Obesity (EASO). EASLEASD-EASO Clinical Practice Guidelines for the Management of Non-Alcoholic Fatty Liver Disease. Obes Facts 2016;9:65-90

59. Chalasani N, Younossi Z, Lavine JE, et al. The diagnosis and management of non-alcoholic fatty liver disease: practice guideline by the American Gastroenterological Association, American Association for the Study of Liver Diseases, and American College of Gastroenterology. Gastroenterology 2012;142:1592-1609.

60. Mózes FE, Lee JA, Selvaraj EA, et al. Diagnostic accuracy of non-invasive tests for advanced fibrosis in patients with NAFLD: an individual patient data meta-analysis. Gut 2022;71:1006-1019.

61. McPherson S, Stewart SF, Henderson E, Burt AD, Day CP. Simple non-invasive fibrosis scoring systems can reliably exclude advanced fibrosis in patients with non-alcoholic fatty liver disease. Gut 2010;59:1265-1269.

62. Torriani FJ, Rodriguez-Torres M, Rockstroh JK, et al. Peginterferon Alfa-2a plus ribavirin for chronic hepatitis $\mathrm{C}$ virus infection in HIV-infected patients. N Engl J Med 2004;351:438-450.

63. Srivastava A, Jong S, Gola A, et al. Cost-comparison analysis of FIB-4, ELF and fibroscan in community pathways for non-alcoholic fatty liver disease. BMC Gastroenterol 2019;19:122.

64. Salva-Pastor N, Chávez-Tapia NC, Uribe M, Nuño-Lámbarri $\mathrm{N}$. The diagnostic and initial approach of the patient with non-alcoholic fatty liver disease: role of the primary care provider. Gastroenterol Hepatol Bed Bench 2019;12:267277.

65. Tapper EB, Krajewski K, Lai M, et al. Simple non-invasive biomarkers of advanced fibrosis in the evaluation of nonalcoholic fatty liver disease. Gastroenterol Rep (Oxf) 2014;2:276-280. 